\title{
Article \\ Convergence Criteria of Three Step Schemes for Solving Equations
}

\author{
Samundra Regmi ${ }^{1}\left(\mathbb{D}\right.$, Christopher I. Argyros ${ }^{2}$, Ioannis K. Argyros ${ }^{3, *(D)}$ and Santhosh George ${ }^{4}$ (D) \\ 1 Learning Commons, University of North Texas at Dallas, Dallas, TX 75201, USA; \\ samundra.regmi@untdallas.edu \\ 2 Department of Computing and Technology, Cameron University, Lawton, OK 73505, USA; \\ christopher.Argyros@cameron.edu \\ 3 Department of Mathematical Sciences, Cameron University, Lawton, OK 73505, USA \\ 4 Department of Mathematical and Computational Sciences, National Institute of Technology Karnataka, \\ Mangalore 575 025, India; sgeorge@nitk.edu.in \\ * Correspondence: iargyros@cameron.edu
}

check for

updates

Citation: Regmi, S.; Argyros, C.I.; Argyros, I.K.; George, S. Convergence Criteria of Three Step Schemes for Solving Equations. Mathematics 2021, 9,3106. https://doi.org/10.3390/ math9233106

Academic Editors: José Manuel Gutiérrez and Ángel Alberto Magreñán

Received: 3 November 2021 Accepted: 29 November 2021 Published: 2 December 2021

Publisher's Note: MDPI stays neutral with regard to jurisdictional claims in published maps and institutional affiliations.

Copyright: (C) 2021 by the authors Licensee MDPI, Basel, Switzerland. This article is an open access article distributed under the terms and conditions of the Creative Commons Attribution (CC BY) license (https:/ / creativecommons.org/licenses/by/ $4.0 /)$.
Abstract: We develop a unified convergence analysis of three-step iterative schemes for solving nonlinear Banach space valued equations. The local convergence order has been shown before to be five on the finite dimensional Euclidean space assuming Taylor expansions and the existence of the sixth derivative not on these schemes. So, the usage of them is restricted six or higher differentiable mappings. But in our paper only the first Frèchet derivative is utilized to show convergence. Consequently, the scheme is expanded. Numerical applications are also given to test convergence.

Keywords: iterative schemes; convergence criterion; Banach space

\section{Introduction}

We study the problem of finding a simple solution $x_{*}$ of

$$
F(x)=0,
$$

provided $F: D \subset E \longrightarrow E_{1}$ is an operator acting between Banach spaces $E$ and $E_{1}$ with $D \neq \varnothing$. Since a closed form solution is not possible in general, iterative schemes are used for solving (1). Many iterative schemes are studied for approximating $x_{*}$. In this paper, we consider the iterative schemes, developed for each $n=0,1,2, \ldots$, as

$$
\begin{aligned}
y_{n} & =x_{n}-F^{\prime}\left(x_{n}\right)^{-1} F\left(x_{n}\right) \\
z_{n} & =y_{n}-A_{n} F^{\prime}\left(x_{n}\right)^{-1} F\left(y_{n}\right) \\
x_{n+1} & =z_{n}-B_{n} F^{\prime}\left(x_{n}\right)^{-1} F\left(z_{n}\right),
\end{aligned}
$$

where $A_{n}=A\left(x_{n}, y_{n}\right), A: D \times D \longrightarrow L\left(E, E_{1}\right), A^{-1} \in L\left(E_{1}, B\right), B_{n}=B\left(x_{n}, y_{n}\right), B$ : $D \times D \longrightarrow L\left(E, E_{1}\right)$ and $B^{-1} \in L\left(E_{1}, E\right)$.

A plethora of schemes are special cases of (2).

Newton's Scheme [1-23]: Set $A_{n}=B_{n}=O$ to obtain

$$
y_{n}=x_{n}-F^{\prime}\left(x_{n}\right)^{-1} F\left(x_{n}\right) \text {. }
$$

This scheme is of order two.

Traub's Scheme [21,24-30]: Set $A_{n}=B_{n}=I$ to obtain

$$
\begin{aligned}
y_{n} & =x_{n}-F^{\prime}\left(x_{n}\right)^{-1} F\left(x_{n}\right) \\
z_{n} & =y_{n}-F^{\prime}\left(x_{n}\right)^{-1} F\left(y_{n}\right) \\
x_{n+1} & =z_{n}-F^{\prime}\left(x_{n}\right)^{-1} F\left(z_{n}\right) .
\end{aligned}
$$


This scheme is of order five.

Ostrowski Scheme [20]: Set $A_{n}=\left(2\left[y_{n}, x_{n} ; F\right]-F^{\prime}\left(x_{n}\right)\right) F^{\prime}\left(x_{n}\right)$ and $B_{n}=O$ to obtain

$$
\begin{aligned}
y_{n} & =x_{n}-F^{\prime}\left(x_{n}\right)^{-1} F\left(x_{n}\right) \\
x_{n+1} & =y_{n}-\left(2\left[y_{n}, x_{n} ; F\right]-F^{\prime}\left(x_{n}\right)\right)^{-1} F\left(y_{n}\right) .
\end{aligned}
$$

This scheme is of order four.

Ostrowski-Type Scheme [20]: Set $A_{n}=\left(\left[y_{n}, x_{n} ; F\right]^{-1}-F^{\prime}\left(x_{n}\right)^{-1}\right) F^{\prime}\left(x_{n}\right)$ and $B_{n}=O$ to obtain

$$
\begin{aligned}
y_{n} & =x_{n}-F^{\prime}\left(x_{n}\right)^{-1} F\left(x_{n}\right) \\
x_{n+1} & =y_{n}-\left(\left[y_{n}, x_{n} ; F\right]^{-1}-F^{\prime}\left(x_{n}\right)^{-1}\right) F\left(y_{n}\right) .
\end{aligned}
$$

This scheme is of order four.

Traub, Newton-type, Runge-Kutta and other three step schemes can be seen as special cases of $(2)[7,14,20,30]$. The local convergence of these schemes and their stability has been given in the aforementioned references using derivatives of order one more than the convergence order. As an example, the existence of the fifth derivative is required for the convergence of the Ostrowski scheme. Notice that convergence can be obtained without the fifth derivative, which does not appear on the scheme. Clearly there is a need to study the convergence of these schemes using a uniform set of criteria. That is why we developed scheme (2).

Let $E=E_{1}=\mathbb{R}, D=[-0.5,1.5]$. Consider $\lambda$ on $D$ by

$$
\lambda(x)=\left\{\begin{array}{cc}
x^{3} \log x^{2}+x^{5}-x^{4} & \text { if } x \neq 0 \\
0 & \text { if } x=0
\end{array}\right.
$$

Then, we get $x_{*}=1$, and

$$
\lambda^{\prime \prime \prime}(x)=6 \log x^{2}+60 x^{2}-24 x+22 .
$$

But, $\lambda^{\prime \prime \prime}(x)$ is unbounded on D. So, the convergence of scheme (2) is not assured by the previous analyses. In order to extend the utilization of these schemes, we study the local convergence of scheme (2): in a Banach space, under generalized continuity conditions and using hypotheses only on the operators appearing on these schemes. Notice also that the convergence of most high convergence order schemes was given only on the finite dimensional Euclidean space with no error bounds on $\left\|x_{n}-x_{*}\right\|$ or information about the uniqueness of the solution. However, we give such results in this paper. Moreover, we present the semi-local convergence of these schemes not given before using majorizing sequences. The technique can apply on other single, multipoint, and multistep schemes along the same lines, since it is so general [8,9,16,27-29].

In Sections 2-4, we present results on majorizing sequences, semi-local and local convergence, respectively. The applications are conclusions can be found in Sections 4 and 5, respectively.

The analysis is provided in the next three sections followed by the examples and conclusions in the last two sections.

\section{Convergence for Majorizing Sequence}

We shall use the following definition of a majorizing sequence.

Definition 1. Let $\left\{\bar{v}_{n}\right\}$ be a sequence in E. Then, a sequence $\left\{v_{n}\right\}$ majorizes $\left\{\bar{v}_{n}\right\}$ if

$$
\left\|\bar{v}_{n+1}-\bar{v}_{n}\right\| \leq v_{n+1}-v_{n} \text { for each } n=0,1,2, \ldots
$$

In this case, the study of the convergence for sequence $\left\{\bar{v}_{n}\right\}$ reduces to that of $\left\{v_{n}\right\}$ [21]. 
Next, we shall define sequences to be shown to be majorizing for scheme (2). Set $T=[0, \infty)$.

Let $a: T \times T \longrightarrow T, \bar{a}: T \times T \longrightarrow T, b: T \times T \times T \longrightarrow T$ and $c: T \times T \times T \times T \longrightarrow T$ be continuous and nondecreasing functions. We denote $a(n)=a_{n}, b(n)=b_{n}$ and $c(n)=c_{n}$. Let $\psi_{0}: T \longrightarrow T$ and $\psi: T_{0} \subseteq T \longrightarrow T$ be continuous and nondecreasing functions for some subset $T_{0}$ of $T$. Let also $\eta \geq 0$. Define scalar sequences $\left\{t_{n}\right\},\left\{s_{n}\right\},\left\{u_{n}\right\}$ by

$$
\begin{aligned}
t_{0} & =0, t_{1}=\eta, \\
u_{n} & =s_{n}+\bar{a}_{n}\left(s_{n}-t_{n}\right), \\
t_{n+1} & =u_{n}+b_{n}\left(u_{n}-s_{n}\right), \\
s_{n+1} & =t_{n+1}+\frac{c_{n}\left(t_{n+1}-u_{n}\right)}{1-\psi_{0}\left(t_{n+1}\right)},
\end{aligned}
$$

where $\bar{\psi}=\left\{\begin{array}{cc}\psi_{0}, & n=0 \\ \psi, & n=1,2, \ldots\end{array}\right.$.

Next, we present two general results on the convergence of majorant sequence (3).

Lemma 1. Suppose that there exists $d>0$ such that for each $n=0,1,2, \ldots$

$$
t_{n} \leq d
$$

and

$$
\psi_{0}(d)<1
$$

Then, sequences $\left\{t_{n}\right\},\left\{s_{n}\right\},\left\{u_{n}\right\}$ converge to their unique least upper bound $t_{*} \in[0, d]$.

Proof. By the definition of these sequences, it follows that they are nondecreasing bounded from above by $d$, with $t_{k} \leq s_{k} \leq u_{k} \leq t_{k+1}$, and as such they converge to $t_{*}$.

Lemma 2. If function $\psi_{0}$ is increasing, then conditions (5) and (6) can be replaced by

$$
t_{n} \leq \psi_{0}^{-1}(1)
$$

Proof. Set $d=\psi_{0}^{-1}(1)$ in Lemma 1.

Remark 1. Convergence criteria (5)-(7) can be verified only in some special cases, since they are very general. That is why we also provide stronger conditions, but easier to be verify.

Define functions $f_{i}, i=1,2,3$ on the interval $[0,1)$ by

$$
\begin{gathered}
f_{1}(t)=a(t) \int_{0}^{1} \psi\left(\theta t^{2} \eta\right) d \theta-t \\
f_{2}(t)=b(t)-t,
\end{gathered}
$$

and

$$
f_{3}(t)=c(t)+t \psi_{0}\left(\frac{\eta}{1-t}\right)-t
$$

Suppose that these functions have smallest zeros $\mu_{i} \in(0,1)$, respectively. Set

$$
\mu=\min \left\{\mu_{i}\right\} \text { and } \mu_{0}=\max \left\{\bar{a}_{0}, b_{0}, \frac{c_{0}}{1-\psi_{0}\left(t_{1}\right)}\right\} .
$$

Then, we can show the third convergence result.

Lemma 3. Suppose that

$$
0 \leq \mu_{0} \leq \mu
$$


Then, sequences $\left\{t_{n}\right\},\left\{s_{n}\right\},\left\{u_{n}\right\}$ are bounded from above by $t_{* *}=\frac{\eta}{1-\mu}$, nondecreasing and converge to $t_{*} \in\left[\eta, t_{* *}\right]$, so that

$$
\begin{gathered}
0 \leq s_{n}-t_{n} \leq \mu\left(t_{n}-s_{n-1}\right) \leq \mu^{2 n} \eta, \\
0 \leq u_{n}-s_{n} \leq \mu\left(s_{n}-t_{n}\right) \leq \mu^{2 n+1} \eta, \\
0 \leq t_{n+1}-u_{n} \leq \mu\left(u_{n}-s_{n}\right) \leq \mu^{2 n+2} \eta,
\end{gathered}
$$

and

$$
0 \leq t_{n+1} \leq \frac{1-\mu^{2 n+3}}{1-\mu} \eta
$$

Proof. Items (10)-(12) hold, provided

$$
\begin{aligned}
& 0 \leq \bar{a}_{k} \leq \mu, \\
& 0 \leq b_{k} \leq \mu,
\end{aligned}
$$

and

$$
0 \leq \frac{c_{k}}{1-\psi_{0}\left(t_{k+1}\right)} \leq \mu
$$

for each $k=0,1,2, \ldots$ However, these items are true for $k=0$, by (9). Let us suppose that (14)-(16) are true for $k=0,1,2, \ldots, n$. By induction hypotheses (10)-(12), we get

$$
\begin{aligned}
t_{k+1} & \leq u_{k}+\mu^{2 k+2} \eta \leq s_{k}+\mu^{2 k+1} \eta+\mu^{2 k+2} \eta \\
& \leq t_{k}+\mu^{2 k} \eta+\mu^{2 k+1} \eta+\mu^{2 k+2} \eta \\
& \leq \eta+\mu \eta+\ldots+\mu^{2 k+2} \eta . \\
& =\frac{1-\mu^{2 k+3}}{1-\mu} \eta \leq \frac{\eta}{1-\mu}=t_{* *} .
\end{aligned}
$$

Then, (14)-(16) shall be true if

$$
f_{i}\left(\mu_{i}\right) \leq 0,
$$

which are true by the definition of $f_{i}$. So, we deduce $\lim _{k \rightarrow \infty} t_{k}=\lim _{k \longrightarrow \infty} s_{k}=\lim _{k \longrightarrow \infty} u_{k}$ $=t_{*}$.

\section{Semi-Local Convergence}

We shall use some functions and parameters.

Suppose that there exists function $\psi_{0}: T \longrightarrow T$ continuous and nondecreasing such that $\psi_{0}(t)-1=0$ has a minimal solution $\rho \in T-\{0\}$. Set $T_{0}=[0, \rho)$. Suppose function $\psi: T_{0} \longrightarrow T$ is continuous and nondecreasing.

The following conditions (A) shall be used.

Suppose:

(A1) There exists $x_{0} \in D, \eta \geq 0$ such that $F^{\prime}\left(x_{0}\right)^{-1} \in L\left(E_{1}, E\right)$ and

$$
\left\|F^{\prime}\left(x_{0}\right)^{-1} F\left(x_{0}\right)\right\| \leq \eta \text {. }
$$

(A2) For each $y \in D$

$$
\left\|F^{\prime}\left(x_{0}\right)^{-1}\left(F^{\prime}(y)-F^{\prime}\left(x_{0}\right)\right)\right\| \leq \psi_{0}\left(\left\|y-x_{0}\right\|\right) .
$$

Set $D_{0}=B\left[x_{0}, s\right] \cap D$, where $B\left(x_{0}, s\right)=\left\{y \in E:\left\|y-x_{0}\right\|<s\right\}$ and $B\left[x_{0}, s\right]$ is the closure of $B\left(x_{0}, s\right)$.

$$
\left\|F^{\prime}\left(x_{0}\right)^{-1}\left(F^{\prime}(w)-F^{\prime}\left(w_{1}\right)\right)\right\| \leq \psi\left(\left\|w-w_{1}\right\|\right) \text { for all } w_{1}, w \in D_{0} .
$$


(A4) For each $n=0,1,2, \ldots$

$$
\begin{gathered}
\left\|A_{n} F^{\prime}\left(x_{n}\right)^{-1} F^{\prime}\left(x_{0}\right)\right\|=\bar{a}_{n} \leq a_{n} \leq a, \\
\left\|B_{n} F^{\prime}\left(x_{n}\right)^{-1} \int_{0}^{1}\left(F^{\prime}\left(y_{n}+\theta\left(z_{n}-y_{n}\right)\right)-F^{\prime}\left(x_{n}\right) A_{n}^{-1}\right) d \theta\right\|=\bar{b}_{n} \leq b_{n} \leq b, \\
\left\|F^{\prime}\left(x_{0}\right)^{-1} \int_{0}^{1}\left(F^{\prime}\left(z_{n}+\theta\left(x_{n+1}-z_{n}\right)\right)-F^{\prime}\left(x_{n}\right) B_{n}^{-1}\right) d \theta\right\|=\bar{c}_{n} \leq c_{n} \leq c .
\end{gathered}
$$

(A5) Conditions of Lemma 1 or Lemma 2 or Lemma 3 hold.

and

(A6) $B\left[x_{0}, t_{*}\right] \subset D\left(\right.$ or $\left.B\left[x_{0}, t_{* *}\right] \subset D\right)$.

The semi-local convergence of scheme (2) is shown next.

Theorem 1. Suppose conditions (A) hold. Then, sequences $\left\{x_{n}\right\},\left\{y_{n}\right\},\left\{z_{n}\right\}$ generated by scheme (2) are well defined in $B\left[x_{0}, t_{*}\right]$, stays in $B\left[x_{0}, t_{*}\right]$ for each $n=0,1,2, \ldots$ and converge to a solution $x_{*} \in B\left[x_{0}, t_{*}\right]$ of equation $F(x)=0$, so that for each $n=0,1,2, \ldots$

$$
\left\|x_{n}-x_{*}\right\| \leq t_{*}-t_{n} .
$$

Proof. We shall prove assertions

$\left(I_{k}\right)\left\|y_{k}-x_{k}\right\| \leq s_{k}-t_{k}$

$\left(I I_{k}\right)\left\|z_{k}-y_{k}\right\| \leq u_{k}-s_{k}$

$\left(I I I_{k}\right)\left\|x_{k+1}-z_{k}\right\| \leq t_{k+1}-u_{k}$ using mathematical induction on $k$. By scheme (2) for $n=0$ and (A1), we have

$$
\left\|y_{0}-x_{0}\right\|=\left\|F^{\prime}\left(x_{0}\right)^{-1} F\left(x_{0}\right)\right\| \leq \eta=s_{0}-t_{0} \leq t_{*},
$$

so $y_{0} \in B\left[x_{0}, t_{*}\right]$ and $\left(I_{0}\right)$ holds. By (A3), (A4) and the second substep of scheme (2), we have

$$
\begin{aligned}
\left\|z_{k}-y_{k}\right\|= & \left\|A_{k} F^{\prime}\left(x_{k}\right)^{-1}\left(F\left(y_{k}\right)-F\left(x_{k}\right)-F^{\prime}\left(x_{k}\right)\left(y_{k}-x_{k}\right)\right)\right\| \\
\leq & \left\|A_{k} F^{\prime}\left(x_{k}\right)^{-1} F^{\prime}\left(x_{0}\right)\right\| \\
& \times\left\|\int_{0}^{1} F^{\prime}\left(x_{0}\right)^{-1}\left(F^{\prime}\left(x_{k}+\theta\left(y_{k}-x_{k}\right)\right)-F^{\prime}\left(x_{k}\right)\right) d \theta\right\|\left\|y_{k}-x_{k}\right\| \\
\leq & \left\|A_{k} F^{\prime}\left(x_{k}\right)^{-1} F^{\prime}\left(x_{0}\right)\right\| \int_{0}^{1} \bar{\psi}\left(\theta\left\|y_{k}-x_{k}\right\|\right) d \theta\left\|y_{k}-x_{k}\right\| \\
\leq & \left\|A_{k} F^{\prime}\left(x_{k}\right)^{-1} F^{\prime}\left(x_{0}\right)\right\| \int_{0}^{1} \bar{\psi}\left(\theta\left(s_{k}-t_{k}\right)\right) d \theta\left(s_{k}-t_{k}\right)=u_{k}-s_{k},
\end{aligned}
$$

showing $\left(I I_{k}\right)$. Similarly by the second substep of scheme (2), we obtain in turn

$$
\begin{aligned}
\left\|x_{k+1}-z_{k}\right\| & =\left\|B_{k} F^{\prime}\left(x_{k}\right)^{-1} F\left(z_{k}\right)\right\| \\
& \leq\left\|B_{k} F^{\prime}\left(x_{k}\right)^{-1} \int_{0}^{1}\left(F^{\prime}\left(y_{k}+\theta\left(z_{k}-y_{k}\right)\right)-F^{\prime}\left(x_{k}\right) A_{k}^{-1}\right) d \theta\right\|\left\|z_{k}-y_{k}\right\| \\
& \left.=\bar{b}_{k}\left\|z_{k}-y_{k}\right\| \leq b_{k} \| u_{k}-s_{k}\right)=t_{k+1}-u_{k}
\end{aligned}
$$


and

$$
\begin{aligned}
\left\|y_{k}-x_{k}\right\| & \leq\left\|F^{\prime}\left(x_{k}\right)^{-1} F^{\prime}\left(x_{0}\right)\right\|\left\|F^{\prime}\left(x_{0}\right)^{-1} F\left(x_{k}\right)\right\| \\
& \leq \frac{\left\|\int_{0}^{1} F^{\prime}\left(x_{0}\right)^{-1}\left(F^{\prime}\left(z_{k-1}+\theta\left(x_{k}-z_{k-1}\right)\right)-F^{\prime}\left(x_{k-1}\right) B_{k-1}^{-1}\right) d \theta\right\|}{1-\psi_{0}\left(\left\|x_{k}-x_{0}\right\|\right)}\left\|x_{k}-z_{k-1}\right\| \\
& =\frac{\bar{c}_{k}\left\|x_{k}-z_{k-1}\right\|}{1-\psi_{0}\left(\left\|x_{k}-x_{0}\right\|\right)} \\
& \leq \frac{c_{k}\left(t_{k}-u_{k-1}\right)}{1-\psi_{0}\left(t_{k}\right)}=s_{k}-t_{k}
\end{aligned}
$$

showing $\left(I_{k}\right)$ and $\left(I I I_{k}\right)$, where we also used

$$
\begin{aligned}
F\left(x_{k}\right) & =F\left(x_{k}\right)-F\left(z_{k-1}\right)+F\left(z_{k-1}\right) \\
& =F\left(x_{k}\right)-F\left(z_{k-1}\right)-F^{\prime}\left(x_{k-1}\right) B_{k-1}^{-1}\left(x_{k}-z_{k-1}\right),
\end{aligned}
$$

(by the third substep of scheme (2))

$$
F\left(z_{k}\right)=F\left(z_{k}\right)-F\left(y_{k}\right)+F\left(y_{k}\right)=F\left(z_{k}\right)-F\left(y_{k}\right)-F^{\prime}\left(x_{k}\right) A_{k}^{-1}\left(z_{k}-y_{k}\right),
$$

(by the second substep of scheme (2)),

$$
\left\|F^{\prime}\left(x_{0}\right)^{-1}\left(F^{\prime}\left(x_{k}\right)-F^{\prime}\left(x_{0}\right)\right)\right\| \leq \psi_{0}\left(\left\|x_{k}-x_{0}\right\|\right) \leq \psi_{0}\left(t_{*}\right)<1,
$$

so

$$
F^{\prime}\left(x_{k}\right)^{-1} F^{\prime}\left(x_{0}\right) \| \leq \frac{1}{1-\psi_{0}\left(\left\|x_{k}-x_{0}\right\|\right)}
$$

by the Banach lemma on invertible operators $[17,20]$. Suppose $\left(I_{k}\right),\left(I I_{K}\right),\left(I I I_{K}\right)$ hold, $y_{k}, z_{k}, x_{k+1} \in B\left[x_{0}, t_{*}\right]$. Then, by repeating these calculations with $x_{k+1}, y_{k+1}, z_{k+1}, x_{k+2}$ replacing $x_{k}, y_{k}, z_{k}, x_{k+1}$, respectively, we complete the induction. Therefore, sequence $\left\{x_{k}\right\}$ is fundamental in a Banach space $E$ so $\lim _{k \rightarrow \infty} x_{k}=x_{*}$ for some $x_{*} \in B\left[x_{0}, t_{*}\right]$. If $k \longrightarrow \infty$ then by the estimate

$$
\left\|F^{\prime}\left(x_{0}\right)^{-1} F\left(x_{k}\right)\right\| \leq c_{k}\left(t_{k+1}-u_{k}\right) \leq c\left(t_{k+1}-u_{k}\right) \longrightarrow 0,
$$

and using the continuity of $F$, we get $F\left(x_{*}\right)=0$.

Next, the uniqueness result of $x_{*}$ is discussed.

\section{Proposition 1. Suppose}

(a) Point $x_{*}$ solves the equation $F(x)=0$.

(b) There exists $\bar{s} \geq t_{*}$ such that

$$
\int_{0}^{1} \psi_{0}\left((1-\theta) \bar{s}+\theta t_{*}\right) d \theta<1
$$

Set $D_{1}=B\left[x_{0}, \bar{s}\right] \cap D$. Then, $x_{*}$ is unique in $D_{1}$.

Proof. Consider $\bar{x} \in D_{1}$ satisfying $F(\bar{x})=0$. Define $M_{0}=\int_{0}^{1} F^{\prime}\left(x_{*}+\theta\left(\bar{x}-x_{*}\right)\right) d \theta$. It then follows from (A1) and (18) that

$$
\begin{aligned}
\left\|F^{\prime}\left(x_{0}\right)^{-1}\left(M_{0}-F^{\prime}\left(x_{0}\right)\right)\right\| & \leq \int_{0}^{1} \psi_{0}\left((1-\theta)\left\|\bar{x}-x_{0}\right\|+\theta\left\|x_{*}-x_{0}\right\|\right) d \theta \\
& \leq \int_{0}^{1} \psi_{0}\left((1-\theta) \bar{s}+\theta t_{*}\right) d \theta<1,
\end{aligned}
$$


so $\bar{x}=x_{*}$ follows from $M_{0}^{-1} \in L\left(E_{1}, E\right)$ and the approximation $M_{0}\left(\bar{x}-x_{*}\right)=F(\bar{x})-$ $F\left(x_{*}\right)=0-0=0$.

Remark 2. We want to see how developed sequences are defined in some interesting cases. RungeKutta and other high convergence schemes can also be studied under this technique by also specializing the operators $A_{n}$ and $B_{n}$. Let us choose $A_{n}=B_{n}=I$. That is, we consider Traub's scheme. Then, we have in turn

$$
\begin{aligned}
\bar{a}_{n} & =\left\|A_{n} F^{\prime}\left(x_{n}\right)^{-1} F^{\prime}\left(x_{0}\right)\right\|=\left\|F^{\prime}\left(x_{n}\right)^{-1} F^{\prime}\left(x_{0}\right)\right\| \\
& \leq \frac{1}{1-\psi_{0}\left(\left\|x_{n}-x_{0}\right\|\right)} \leq \frac{1}{1-\psi_{0}\left(t_{n}\right)}=: a_{n} \\
\bar{b}_{n} & =\| F^{\prime}\left(x_{n}\right)^{-1}\left(\int_{0}^{1}\left(F^{\prime}\left(y_{n}+\theta\left(z_{n}-y_{n}\right)\right)-F^{\prime}\left(z_{n}\right)\right) d \theta+\left(F^{\prime}\left(z_{n}\right)-F^{\prime}\left(x_{n}\right)\right) \|\right. \\
& \leq \frac{\left.\int_{0}^{1} \psi\left((1-\theta) \| z_{n}-y_{n}\right)\right) d \theta+\psi\left(\theta\left\|z_{n}-x_{n}\right\|\right)}{1-\psi_{0}\left(\left\|x_{n}-x_{0}\right\|\right)} \\
& \leq \frac{\int_{0}^{1} \psi\left((1-\theta)\left(u_{n}-s_{n}\right)\right) d \theta+\psi\left(u_{n}-t_{n}\right)}{1-\psi_{0}\left(t_{n}\right)}=: b_{n}, \\
\bar{c}_{n} \leq & \| F^{\prime}\left(x_{0}\right)^{-1}\left(\int_{0}^{1}\left(F^{\prime}\left(z_{n-1}+\theta\left(x_{n}-z_{n-1}\right)\right)-F^{\prime}\left(x_{n}\right)\right) d \theta+\left(F^{\prime}\left(x_{n}\right)-F^{\prime}\left(x_{n-1}\right)\right) \|\right. \\
\leq & \frac{\left.\int_{0}^{1} \psi\left((1-\theta) \| x_{n}-z_{n-1}\right)\right) d \theta+\psi\left(\left\|x_{n}-x_{n-1}\right\|\right)}{1-\psi_{0}\left(\left\|x_{n-1}-x_{0}\right\|\right)} \\
\leq & \frac{\int_{0}^{1} \psi\left((1-\theta)\left(t_{n}-u_{n-1}\right)\right) d \theta+\psi\left(t_{n}-t_{n-1}\right)}{1-\psi_{0}\left(t_{n-1}\right)}=: c_{n-1} .
\end{aligned}
$$

Under these choices, we shall present another result on majorizing sequences using our idea of recurrent functions, which is weaker than that of Lemma 3. We have in this case

$$
\begin{gathered}
\bar{a}_{n}=\frac{\int_{0}^{1} \bar{\psi}\left((1-\theta)\left(s_{n}-t_{n}\right)\right) d \theta}{1-\psi_{0}\left(t_{n}\right)}, \\
b_{n}=\frac{\int_{0}^{1} \psi\left((1-\theta)\left(u_{n}-s_{n}\right)\right) d \theta+\psi\left(\left(u_{n}-s_{n}\right)+\left(s_{n}-t_{n}\right)\right)}{1-\psi_{0}\left(t_{n}\right)}
\end{gathered}
$$

and

$$
\frac{c_{n}}{1-\psi_{0}\left(t_{n+1}\right)}=\frac{\int_{0}^{1} \psi\left((1-\theta)\left(t_{n+1}-u_{n}\right)\right) d \theta+\psi\left(\left(t_{n+1}-u_{n}\right)+\left(u_{n}-s_{n}\right)+\left(s_{n}-t_{n}\right)\right)}{1-\psi_{0}\left(t_{n+1}\right)} .
$$

Let us consider the interesting case when $\psi_{0}(t)=L_{0}$ and $\psi(t)=L t$. 
Define sequences of functions $\left\{h_{n}^{(j)}\right\}, j=1,2,3$ on the interval $[0,1)$ by

$$
\begin{aligned}
h_{n}^{(1)}= & \frac{L}{2} t^{2 n-1} \eta+L_{0}\left(1+t+\ldots+t^{2 n}\right) \eta-1, \\
h_{n}^{(2)}(t)= & \frac{L}{2} t^{2 n} \eta+L\left(t^{2 n}+t^{2 n-1}\right) \eta+L_{0}\left(1+t+\ldots+t^{2 n}\right) \eta-1, \\
h_{n}^{(3)}= & \frac{L}{2} t^{2 n+1} \eta+L\left(t^{2 n+1}+t^{2 n}+t^{2 n-1}\right) \eta \\
& +L_{0}\left(1+t+\ldots+t^{2 n}\right) \eta-1, \\
g_{1}(t)= & L_{0} t^{3}+\left(L_{0}+\frac{L}{2}\right) t^{2}-\frac{L}{2} \\
g_{2}(t)= & \left(\frac{3 L}{2}+L_{0}\right) t^{3}+\left(L_{0}+L\right) t^{2}-\frac{3}{2} L t-L, \\
g_{3}(t)= & L_{0} t^{5}+\left(\frac{3 L}{2}+L_{0}\right) t^{4}+L t^{3}-\frac{L}{2} t^{2}-L t-L, \\
h_{\infty}^{(1)}(t)= & h_{\infty}^{(2)}(t)=h_{\infty}^{(3)}(t)=\frac{L \eta}{1-t}-1 .
\end{aligned}
$$

Notice that $g_{1}(0)=-\frac{L}{2}, g_{2}(0)=g_{3}(0)=-L, g_{1}(1)=g_{2}(1)=g_{3}(1)=2 L_{0}$. It then follows (by the intermediate value theorem) that polynomial $g_{j}$ have zeros in $(0,1)$. Call by $\rho_{j}$ the minimal such zeros, respectively. Set $\rho=\min \left\{\rho_{j}\right\}$.

Next, we show another result on majorizing sequences for scheme (2).

Lemma 4. Suppose that

$$
\mu_{0} \leq \rho<1-L_{0} \eta .
$$

Then, the conclusions of Lemma 3 hold for sequences $\left\{s_{n}\right\},\left\{t_{n}\right\},\left\{u_{n}\right\}$ with $\rho$ replacing $\mu$.

Proof. We shall show this time

$$
\begin{gathered}
0 \leq \bar{a}_{k} \leq \rho, \\
0 \leq b_{k} \leq \rho, \\
0 \leq \frac{c_{k}}{1-L_{0} t_{k+1}} \leq \rho
\end{gathered}
$$

and

$$
t_{k} \leq s_{k} \leq u_{k} \leq t_{k+1}
$$

These assertions hold for $k=0$ by (48) and the definition of these sequences (see Remark 2). Then, as in Lemma 3, we can show instead of (20) that

$$
\frac{L}{2} \rho^{2 k-1} \eta+L_{0}\left(1+\rho+\ldots+\rho^{2 k}\right) \eta-1 \leq 0 .
$$

This estimate motivates us to define recurrent functions $h_{k}^{(1)}$ and show instead

$$
h_{k}^{(1)}(\rho) \leq 0 .
$$

We need a relationship between two consecutive functions $h_{K}^{(1)}$. Then, we can write

$$
\begin{aligned}
h_{k+1}^{(1)}= & \frac{L}{2} t^{2 k+1} \eta+L_{0}\left(1+t+\ldots+t^{2 k+2}\right) \eta-1 \\
& -\frac{L}{2} t^{2 k-1}-L_{0}\left(1+t+\ldots+t^{2 k}\right) \eta+1+h_{k}^{(1)}(t) \\
= & h_{k}^{(1)}(t)+g_{1}(t) t^{2 k-1} \eta .
\end{aligned}
$$


In particular, we have

$$
h_{k+1}^{(1)}\left(\rho_{1}\right)=h_{k}\left(\rho_{1}\right)
$$

by the definition of $\rho_{1}$. Define also function $h_{\infty}^{(1)}(t)=\lim _{k \rightarrow \infty} h_{k}^{(1)}(t)$. Then, we have

$$
h_{\infty}^{(1)}(t)=\frac{L_{0} \eta}{1-t}-1
$$

Hence, we can show instead of (20) that

$$
h_{\infty}^{(1)}\left(\rho_{1}\right) \leq 0,
$$

which is true by (48). The induction for (20) is completed. Moreover, similarly instead of (21) we can show that

$$
\frac{L}{2} \rho^{2 k} \eta+L\left(\rho^{2 k}+\rho^{2 k-1}\right) \eta+L_{0}\left(1+\rho+\ldots+\rho^{2 k}\right) \eta-1 \leq 0
$$

or

$$
h_{k}^{(2)}(\rho) \leq 0
$$

Then, again we have

$$
\begin{aligned}
h_{k+1}^{(2)}= & \frac{L}{2} t^{2 k+2} \eta+L\left(t^{2 k+2}+t^{2 k+1}\right) \eta \\
& +L_{0}\left(1+t+\ldots+t^{2 k+2}\right) \eta-1 \\
& -\frac{L}{2} t^{2 k} \eta-\frac{L}{2} t^{2 k} \eta-L\left(t^{2 k}+t^{2 k-1}\right) \eta \\
& -L_{0}\left(1+t+\ldots+t^{2 k}\right) \eta+1+h_{k}^{(2)}(t) \\
= & h_{k}^{(2)}(t)+g_{2}(t) t^{2 k-1} \eta .
\end{aligned}
$$

We have in particular

$$
h_{k+1}^{(2)}\left(\rho_{2}\right)=h_{k}^{(2)}\left(\rho_{2}\right) \text {. }
$$

Define function $h_{\infty}^{(2)}(t)=\lim _{k \longrightarrow \infty} h_{k}^{(2)}(t)$. Then, we have

$$
h_{\infty}^{(2)}(t)=h_{\infty}^{(1)}(t) .
$$

Hence, we can show instead of (30) that $h_{\infty}^{(2)}\left(\rho_{2}\right) \leq 0$, which is true by (48). Furthermore, (22) holds if we show instead

$$
\frac{L}{2} \rho^{2 k+1} \eta+L\left(\rho^{2 k+1}+\rho^{2 k}+\rho^{2 k-1}\right) \eta+L_{0}\left(1+\rho+\ldots+\rho^{2 k+2}\right) \eta-1 \leq 0
$$

or

$$
h_{k}^{(3)}(\rho) \leq 0
$$

However, we get

$$
\begin{aligned}
h_{k+1}^{(3)}= & \frac{L}{2} t^{2 k+3} \eta+L\left(t^{2 k+3}+t^{2 k+2}+t^{2 k+1}\right) \eta \\
& +L_{0}\left(1+t+\ldots+t^{2 k+4}\right) \eta-1 \\
& -\frac{L}{2} t^{2 k+1} \eta-L\left(t^{2 k+1}+t^{2 k}+t^{2 k-1}\right) \eta \\
& -L_{0}\left(1+t+\ldots+t^{2 k+2}\right) \eta+1+h_{k}^{(3)}(t) \\
= & h_{k}^{(3)}(t)+g_{3}(t) t^{2 k-1} \eta .
\end{aligned}
$$


In particular, we have

$$
h_{k+1}^{(3)}\left(\rho_{3}\right)=h_{k}^{(3)}\left(\rho_{3}\right) \text {. }
$$

Defined function $h_{\infty}^{(3)}(t)=\lim _{k \longrightarrow \infty} h_{k}^{(3)}(t)$. Then, we also get

$$
h_{\infty}^{(3)}(t)=h_{\infty}^{(1)}(t) \text {. }
$$

Hence, (33) holds if $h_{\infty}^{(3)}\left(\rho_{3}\right) \leq 0$. However, this is true by (48). Therefore, the induction is terminated for (20)-(23). The rest is as in the proof of Lemma 1.

\section{Local Convergence}

We shall introduce real parameter and functions to be used in the convergence analysis. Set $M=[0, \infty)$.

Suppose

(i) $\quad \varphi_{0}(t)-1$ has a minimal zero $R_{0} \in M-\{0\}$, where $\varphi_{0}: M \longrightarrow M$ is some continuous and nondecreasing function. Let $M_{0}=\left[0, R_{0}\right)$.

(ii) $\varphi_{1}(t)-1$ has a minimal zero $R_{1} \in M_{0}-\{0\}$, where function $\varphi: M_{0} \longrightarrow M$ is continuous and nondecreasing and $\varphi_{1}: M_{0} \longrightarrow M$ is defined by

$$
\varphi_{1}(t)=\frac{\int_{0}^{1} \varphi((1-\theta) t) d \theta}{1-\varphi_{0}(t)} .
$$

(iii) $\varphi_{0}\left(\varphi_{1}(t) t\right)-1$ has a minimal zero $\bar{R}_{1} \in M_{0}-\{0\}$. Let $\bar{R}_{2}=\min \left\{R_{0}, \bar{R}_{1}\right\}$ and $M_{1}=\left[0, \bar{R}_{2}\right)$.

(iv) $\varphi_{2}(t)$ - has a smallest zero $R_{2} \in M_{1}-\{0\}$, where

$$
\varphi_{2}(t)=\left[\varphi_{1}\left(\varphi_{1}(t) t\right)+\frac{\left(\varphi_{0}(t)+h\left(t, \varphi_{1}(t) t\right)\right) \int_{0}^{1} \omega\left(\theta \varphi_{1}(t) t\right) d \theta}{\left(1-\varphi_{0}(t)\right)\left(1-\varphi_{0}\left(\varphi_{1}(t) t\right)\right)}\right] \varphi_{1}(t),
$$

with functions $\omega: M_{1} \longrightarrow M$ and $h: M \times M_{1} \longrightarrow M$ are continuous and nondecreasing.

(v) $\varphi_{3}(t)-1$ has a smallest zero $R_{3} \in M_{1}-\{0\}$ where

$$
\varphi_{3}(t)=\left[\varphi_{1}\left(\varphi_{2}(t) t\right)+\frac{\left(\varphi_{0}(t)+h_{1}\left(t, \varphi_{1}(t) t, \varphi_{2}(t) t\right)\right) \int_{0}^{1} \omega\left(\theta \varphi_{2}(t) t\right) d \theta}{\left(1-\varphi_{0}(t)\right)\left(1-\varphi_{0}\left(\varphi_{2}(t) t\right)\right)}\right] \varphi_{2}(t),
$$

where $h_{1}: M \times M_{1} \times M_{1} \longrightarrow M$ is nondecreasing and continuous. It shall be shown that

$$
R=\min \left\{R_{i}\right\}, i=1,2,3
$$

is a convergence radius for scheme (2). Set $M_{2}=[0, R)$. These definitions imply that for each $t \in E_{2}$

$$
\begin{gathered}
0 \leq \varphi_{0}(t)<1, \\
0 \leq \varphi_{0}\left(\varphi_{1}(t) t\right)<1,
\end{gathered}
$$

and

$$
0 \leq \varphi_{1}(t)<1, i=1,2,3 .
$$

The conditions $(\mathrm{H})$ shall be used.

Suppose

(H1) For all $w \in D$

$$
\left\|F^{\prime}\left(x_{*}\right)^{-1}\left(F^{\prime}(w)-F^{\prime}\left(x_{*}\right)\right)\right\| \leq \varphi_{0}\left(\left\|w-x_{*}\right\|\right) .
$$

Let $D_{0}=B\left(x_{*}, R_{0}\right) \cap D$. 
(H2) For all $w, w_{1}, z \in D_{0}$

$$
\begin{gathered}
\left\|F^{\prime}\left(x_{*}\right)^{-1}\left(F^{\prime}\left(w_{1}\right)-F^{\prime}(w)\right)\right\| \leq \varphi\left(\left\|w_{1}-w\right\|\right), \\
\left\|F^{\prime}\left(x_{*}\right)^{-1} F^{\prime}(w)\right\| \leq \omega\left(\left\|w-x_{*}\right\|\right), \\
\left\|F^{\prime}\left(x_{*}\right)^{-1}\left(F^{\prime}\left(x_{*}\right)-F^{\prime}\left(w_{1}\right) A\left(w, w_{1}\right)\right)\right\| \leq h\left(\left\|w-x_{*}\right\|,\left\|w_{1}-x_{*}\right\|\right)
\end{gathered}
$$

and

$$
\left\|F^{\prime}\left(x_{*}\right)^{-1}\left(F^{\prime}\left(x_{*}\right)-F^{\prime}(z) B\left(w, w_{1}\right)\right)\right\| \leq h_{1}\left(\left\|w-x_{*}\right\|,\left\|w_{1}-x_{*}\right\|,\left\|z-x_{*}\right\|\right)
$$

(H3) $B\left[x_{*}, R\right] \subset D$.

The local convergence of scheme (2) is given next based on the previous notation and conditions $(\mathrm{H})$.

Theorem 2. Suppose: $x_{0} \in B\left(x_{*}, R\right)-\left\{x_{*}\right\}$ and conditions $(H)$ hold. Then, we conclude $\lim _{n \longrightarrow \infty} x_{n}=\lim _{n \longrightarrow \infty} y_{n}=\lim _{n} \longrightarrow \infty z_{n}=x_{*}$.

Proof. Let $v \in B\left(x_{*}, R\right)-\left\{x_{*}\right\}$. Using (17), (18) and (H1), we have

$$
\left\|F^{\prime}\left(x_{*}\right)^{-1}\left(F^{\prime}(v)-F^{\prime}\left(x_{*}\right)\right)\right\| \leq \varphi_{0}\left(\left\|v-x_{*}\right\|\right) \leq \varphi_{0}(R)<1,
$$

so

$$
\left\|F^{\prime}(v)^{-1} F^{\prime}\left(x_{*}\right)\right\| \leq \frac{1}{1-\varphi_{0}\left(\left\|v-x_{*}\right\|\right)} .
$$

In particular, iterate is well defined by (38) for $v=x_{0}$ and the first substep of scheme (2), from which we can also write

$$
\begin{aligned}
y_{0}-x_{*}= & x_{0}-x_{*}-F^{\prime}\left(x_{0}\right)^{-1} F\left(x_{0}\right) \\
= & \left(F^{\prime}\left(x_{0}\right)^{-1} F^{\prime}\left(x_{*}\right)\right) \\
& \times \int_{0}^{1} F^{\prime}\left(x_{*}\right)^{-1}\left(F^{\prime}\left(x_{*}+\theta\left(x_{0}-x_{*}\right)\right)-F^{\prime}\left(x_{0}\right)\right) d \theta\left(x_{0}-x_{*}\right) .
\end{aligned}
$$

By (34), (37) (for $i=1)$, (38) (for $\left.v=x_{0}\right)$, (39) and (H2), we find in turn that

$$
\begin{aligned}
\left\|y_{0}-x_{*}\right\| & \leq \frac{\int_{0}^{1} \bar{\varphi}\left((1-\theta)\left\|x_{0}-x_{*}\right\|\right) d \theta\left\|x_{0}-x_{*}\right\|}{1-\varphi_{0}\left(\left\|x_{0}-x_{*}\right\|\right)} \\
& \leq \varphi_{1}\left(\left\|x_{0}-x_{*}\right\|\right)\left\|x_{0}-x_{*}\right\| \\
& \leq\left\|x_{0}-x_{*}\right\|<R
\end{aligned}
$$

so $y_{0} \in B\left(x_{*}, R\right)$, where $\bar{\varphi}=\left\{\begin{array}{cc}\varphi_{0}, & k=0 \\ \varphi, & k=1,2, \ldots\end{array}\right.$. We also have that (38) holds for $v=y_{0}$, and iterate $z_{0}$ is well defined, from which we can write in turn that

$$
\begin{aligned}
z_{0}-x_{*}= & y_{0}-x_{*}-F^{\prime}\left(y_{0}\right)^{-1} F\left(y_{0}\right) \\
& +\left(F^{\prime}\left(y_{0}\right)^{-1}-A_{0} F^{\prime}\left(x_{0}\right)^{-1}\right) F\left(y_{0}\right) \\
= & y_{0}-x_{*}-F^{\prime}\left(y_{0}\right)^{-1} F\left(y_{0}\right) \\
& +F^{\prime}\left(y_{0}\right)^{-1}\left(F^{\prime}\left(x_{0}\right)-F^{\prime}\left(y_{0}\right) A_{0}\right) F^{\prime}\left(x_{0}\right)^{-1} F\left(y_{0}\right) .
\end{aligned}
$$

In view of (34), (37) (for $i=2)$, (38) (for $\left.v=x_{0}, y_{0}\right),(40)$, (41) and (H2), we obtain in turn 


$$
\begin{aligned}
&\left\|z_{0}-x_{*}\right\| \leq {\left[\varphi_{1}\left(\varphi_{1}\left(\left\|x_{0}-x_{*}\right\|\right)\left\|x_{0}-x_{*}\right\|\right)\right.} \\
&+\left.\frac{\left.\left(\varphi_{0}\left(\left\|x_{0}-x_{*}\right\|\right)+h\left(\left\|x_{0}-x_{*}\right\|,\left\|y_{0}-x_{*}\right\|\right)\right) \int_{0}^{1} \omega\left(\theta\left\|y_{0}-x_{*}\right\|\right) d \theta\right]}{\left(1-\varphi_{0}\left(\left\|y_{0}-x_{*}\right\|\right)\right)\left(1-\varphi_{0}\left(\left\|x_{0}-x_{*}\right\|\right)\right)}\right]\left\|y_{0}-x_{*}\right\| \\
& \leq \quad \varphi_{2}\left(\left\|x_{0}-x_{*}\right\|\right)\left\|x_{0}-x_{*}\right\| \leq\left\|x_{0}-x_{*}\right\|<R, \\
& \text { so } z_{0} \in B\left(x_{*}, R\right) . \text { Similarly, but using the third substep of scheme (2), we can write } \\
& x_{1}-x_{*}= z_{0}-x_{*}-F^{\prime}\left(z_{0}\right)^{-1} F\left(z_{0}\right) \\
&+\left(F^{\prime}\left(z_{0}\right)^{-1}-B_{0} F^{\prime}\left(x_{0}\right)^{-1}\right) F\left(z_{0}\right) \\
&= z_{0}-x_{*}-F^{\prime}\left(z_{0}\right)^{-1} F\left(z_{0}\right) \\
&+\left(F^{\prime}\left(z_{0}\right)^{-1}-B_{0} F^{\prime}\left(x_{0}\right)^{-1}\right) F\left(z_{0}\right) \\
&= z_{0}-x_{*}-F^{\prime}\left(z_{0}\right)^{-1} F\left(z_{0}\right)+F^{\prime}\left(z_{0}\right)^{-1}\left(\left(F^{\prime}\left(x_{0}\right)-F^{\prime}\left(x_{*}\right)\right)\right. \\
&\left.+\left(F^{\prime}\left(x_{*}\right)-F^{\prime}\left(z_{0}\right) B_{0}\right)\right) F^{\prime}\left(x_{0}\right)^{-1} F\left(z_{0}\right),
\end{aligned}
$$

so

$$
\begin{aligned}
\left\|x_{1}-x_{*}\right\| \leq & {\left[\varphi_{1}\left(\varphi_{2}\left(\left\|x_{0}-x_{*}\right\|\right)\left\|x_{0}-x_{*}\right\|\right)\right.} \\
& \left.+\frac{H}{\left(1-\varphi_{0}\left(\varphi_{2}\left(\left\|x_{0}-x_{*}\right\|\right)\left\|x_{0}-x_{*}\right\|\right)\right)\left(1-\varphi_{0}\left(\left\|x_{0}-x_{*}\right\|\right)\right)}\right] \\
& \times \varphi_{2}\left(\left\|x_{0}-x_{*}\right\|\right) \\
\leq & \varphi_{3}\left(\left\|x_{0}-x_{*}\right\|\right)\left\|x_{0}-x_{*}\right\| \leq\left\|x_{0}-x_{*}\right\|,
\end{aligned}
$$

for $H=\left(\varphi_{0}\left(\left\|x_{0}-x_{*}\right\|\right)+h_{1}\left(\left\|x_{0}-x_{*}\right\|, \varphi_{1}\left(\left\|x_{0}-x_{*}\right\|\right)\left\|x_{0}-x_{*}\right\|, \varphi_{2}\left(\left\|x_{0}-x_{*}\right\|\right) \| x_{0}-\right.\right.$ $\left.\left.x_{*} \|\right)\right) \int_{0}^{1} \omega\left(\theta \varphi_{2}\left(\left\|x_{0}-x_{*}\right\|\right)\left\|x_{0}-x_{*}\right\|\right) d \theta$. Simply, switch $x_{0}, y_{0}, z_{0}, x_{1}$ by $x_{k}, y_{k}, z_{k}, x_{k+1}$, respectively, in the preceding calculations to get

$$
\begin{aligned}
\left\|y_{k}-x_{*}\right\| & \leq \varphi_{1}\left(\left\|x_{k}-x_{*}\right\|\right)\left\|x_{k}-x_{*}\right\| \leq\left\|x_{k}-x_{*}\right\|<R \\
\left\|z_{k}-x_{*}\right\| & \leq \varphi_{2}\left(\left\|x_{k}-x_{*}\right\|\right)\left\|x_{k}-x_{*}\right\| \leq\left\|x_{k}-x_{*}\right\|<R
\end{aligned}
$$

and

$$
\left\|x_{k+1}-x_{*}\right\| \leq \varphi_{3}\left(\left\|x_{k}-x_{*}\right\|\right)\left\|x_{k}-x_{*}\right\| \leq\left\|x_{k}-x_{*}\right\| .
$$

Then, from the estimation

$$
\left\|x_{k+1}-x_{*}\right\| \leq d\left\|x_{k}-x_{*}\right\|<R,
$$

where $d=\varphi_{3}\left(\left\|x_{k}-x_{*}\right\|\right)$, we get $x_{k+1} \in B\left(x_{*}, R\right)$ and $\lim _{k \rightarrow \infty} x_{k}=x_{*}$.

A uniqueness result is given next.

\section{Proposition 2. Suppose:}

(i) Point $x_{*}$ is a simple solution of equation $F(x)=0$.

(ii)

$$
\int_{0}^{1} \varphi_{0}\left(\theta R_{*}\right) d \theta<1, \text { for some } R_{*} \geq R .
$$

Set $D_{1}=B\left[x_{*}, R_{*}\right] \cap D$. Then, $x_{*}$ is unique in $D_{1}$. 
Proof. Consider $\tilde{x} \in D_{1}$ with $F(\tilde{x})=0$. Set $S=\int_{0}^{1} F^{\prime}\left(x_{*}+\theta\left(\tilde{x}-x_{*}\right)\right) d \theta$. Using (H1) and (47), we obtain

$$
\left\|F^{\prime}\left(x_{*}\right)^{-1}\left(S-F^{\prime}\left(x_{*}\right)\right)\right\| \leq \int_{0}^{1} \varphi_{0}\left(\theta \| \tilde{x}-x_{*}\right) d \theta \leq \int_{0}^{1} \varphi_{0}\left(\theta R_{*}\right) d \theta<1,
$$

so $\tilde{x}=x_{*}$ follows by $S^{-1} \in L\left(E_{1}, E\right)$ and $S\left(\tilde{x}-x_{*}\right)=F(\tilde{x})-F\left(x_{*}\right)=0-0=0$.

\section{Numerical Experiments}

We consider four examples with $A_{n}=B_{n}=I$ (Multistep Newton method). Here, $\varphi_{0}(t)=a_{0} t, \varphi(t)=a t, \omega(t)=a_{1} t$ and $h(t, t)=h_{1}(t, t, t)=\varphi_{0}(t)$.

Example 1. Define function

$$
q(t)=\delta_{0} t+\delta_{1}+\delta_{2} \sin \delta_{3} t, t_{0}=0,
$$

where $\delta_{j}, j=0,1,2,3$ are numbers. Then, for $\delta_{3}$ large and $\delta_{2}$ small, $\frac{a_{0}}{a}$ is arbitrarily small and $\frac{a_{0}}{a} \longrightarrow 0$.

Example 2. If $E=E_{1}=C[0,1], D=B[0,1]$, consider $Q: D \longrightarrow E_{1}$ given as

$$
Q(\lambda)(x)=\varphi(x)-5 \int_{0}^{1} x \theta \lambda(\theta)^{3} d \theta .
$$

We obtain

$$
Q^{\prime}(\lambda(\xi))(x)=\xi(x)-15 \int_{0}^{1} x \theta \lambda(\theta)^{2} \xi(\theta) d \theta, \text { for each } \xi \in D,
$$

so for $x_{*}=0$, we set $a_{0}=7.5, a=a_{4}=K=15, a_{1}=\frac{L_{0}}{2}$. Then, the radii:

$$
r_{1}=0.066667=r, r_{2}=0.109818, \text { and } \rho_{R}=\frac{2}{3 K}=0.0667 .
$$

Example 3. Consider the motion system

$$
N_{1}^{\prime}\left(w_{1}\right)=e^{w_{1}}, N_{2}^{\prime}\left(w_{2}\right)=(e-1) w_{2}+1, N_{3}^{\prime}\left(w_{3}\right)=1
$$

with $N_{1}(0)=N_{2}(0)=N_{3}(0)=0$. Let $N=\left(N_{1}, N_{2}, N_{3}\right)$. Let $E=E_{1}=\mathbb{R}^{3}, D=B[0,1], x_{*}=$ $(0,0,0)^{T}$. Let function $N$ on $D$ for $w=\left(w_{1}, w_{2}, w_{3}\right)^{T}$ given as

$$
N(w)=\left(e^{w_{1}}-1, \frac{e-1}{2} w_{2}^{2}+w_{2}, w_{3}\right)^{T} .
$$

Then, $N^{\prime}$ is given as

$$
N^{\prime}(w)=\left[\begin{array}{ccc}
e^{x} & 0 & 0 \\
0 & (e-1) w_{2}+1 & 0 \\
0 & 0 & 1
\end{array}\right] .
$$

Then, we can set $a_{0}=(e-1), a=e^{\frac{1}{e-1}}=a_{4}, a_{1}=\frac{a_{0}}{2}$ and $K=e$. Then, the radii:

$$
r_{1}=0.382692=r, r_{2}=0.417923, \text { and } \rho_{R}=\frac{2}{3 K}=0.2453,
$$

where $K$ is the Lipschitz constant in D given in [26,30].

Example 4. Consider E, $E_{1}$ and $D$ as in the second Example. Then, the (BVP) [13]

$$
\begin{gathered}
p(0)=0, p(1)=1, \\
p^{\prime \prime}=-p-\sigma p^{2}
\end{gathered}
$$


can be also given as

$$
p(s)=s+\int_{0}^{1} G(s, t)\left(p^{3}(t)+\sigma p^{2}(t)\right) d t
$$

where $\sigma$ is a constant, Consider $F: D \longrightarrow V_{2}$ as

$$
[F(x)](s)=x(s)-s-\int_{0}^{1} G(s, t)\left(x^{3}(t)+\sigma x^{2}(t)\right) d t .
$$

Let us set $p_{0}(s)=s$ and $D=B\left(p_{0}, \rho_{0}\right)$. Then, clearly $B\left(p_{0}, \rho_{0}\right) \subset B\left(0, \rho_{0}+1\right)$, since $\left\|p_{0}\right\|=1$. Suppose $2 \sigma<5$. Then, conditions $(A)$ are satisfied for

$$
a_{0}=\frac{2 \sigma+3 \rho_{0}+6}{8}, a=\frac{\sigma+6 \rho_{0}+3}{4},
$$

$a_{1}=\frac{a_{0}}{2}$ and $\mu=\frac{1+\sigma}{5-2 \sigma}$. Notice that $a_{0}<a_{1}$.

\section{Conclusions}

We have provided a single sufficient criterion for the semi-local convergence of three step schemes. Upon specializing the parameters involved we show that although our majorizing sequence is more general than earlier ones: Convergence criteria are weaker (i.e., the utility of the schemes is extended); the upper error estimates are more accurate (i.e., at least as few iterates are required to achieve a predecided error tolerance) and we have an at least as large ball containing the solution. These benefits are obtained without additional hypotheses. According to our new technique we locate a more accurate domain than before containing the iterates resulting to more accurate (at least as small) Lipschitz condition.

Our theoretical results are further justified using numerical experiments. In the future we plan to extend these results by replacing the Lipschitz constants by generalized functions along the same lines.

Author Contributions: Conceptualization, S.R., C.I.A., I.K.A. and S.G.; software, S.R., C.I.A., I.K.A. and S.G.; validation, S.R., C.I.A., I.K.A. and S.G.; formal analysis, S.R., C.I.A., I.K.A. and S.G.; investigation, S.R., C.I.A., I.K.A. and S.G.; resources, S.R., C.I.A., I.K.A. and S.G.; data curation, S.R., C.I.A., I.K.A. and S.G.; writing —original draft preparation, S.R., C.I.A., I.K.A. and S.G.; writing-review and editing, S.R., C.I.A., I.K.A. and S.G.; visualization, S.R., C.I.A., I.K.A. and S.G.; supervision, S.R., C.I.A., I.K.A. and S.G.; project administration, S.R., C.I.A., I.K.A. and S.G.; funding acquisition, S.R., C.I.A., I.K.A. and S.G. All authors have read and agreed to the published version of the manuscript.

Funding: This research received no external funding.

Conflicts of Interest: The authors declare no conflict of interest.

\section{References}

1. Argyros, I.K. On the Newton-Kantorovich hypothesis for solving equations. J. Comput. Math. 2004, 169, 315-332. [CrossRef]

2. Argyros, I.K. Computational Theory of Iterative Schemes; Studies in Computational Mathematics Series, 15; Chui, C.K., Wuytack, L., Eds.; Elsevier Publ. Co.: New York, NY, USA, 2007.

3. Argyros, I.K. Convergence and Applications of Newton-Type Iterations; Springer: Berlin, Germany, 2008.

4. Argyros, I.K.; Hilout, S. Weaker conditions for the convergence of Newton's scheme. J. Complex. 2012, 28, 364-387. [CrossRef]

5. Argyros, I.K.; Hilout, S. On an improved convergence analysis of Newton's scheme. Appl. Math. Comput. 2013, 225, 372-386.

6. Argyros, I.K.; Magréñan, A.A. Iterative Schemes and Their Dynamics with Applications; CRC Press: New York, NY, USA, 2017.

7. Argyros, I.K.; Magréñan, A.A. A Contemporary Study of Iterative Schemes; Elsevier: New York, NY, USA, 2018.

8. Behl, R.; Maroju, P.; Martinez, E.; Singh, S. A study of the local convergence of a fifth order iterative scheme. Indian J. Pure Appl. Math. 2020, 51, 439-455.

9. Cătinaş, E. The inexact, inexact perturbed, and quasi-Newton schemes are equivalent models. Math. Comput. 2005, 74, 291-301. [CrossRef]

10. Chen, X.; Yamamoto, T. Convergence domains of certain iterative schemes for solving nonlinear equations. Numer. Funct. Anal. Optim. 1989, 10, 37-48. [CrossRef] 
11. Dennis, J.E., Jr.; Schnabel, R.B. Numerical Schemes for Unconstrained Optimization and Nonlinear Equations; SIAM: Philadelphia, PA, USA, 1996; First published by Prentice-Hall: Englewood Cliffs, NJ, USA, 1983.

12. Deuflhard, P. Newton Schemes for Nonlinear Problems. Affine Invariance and Adaptive Algorithms; Springer Series in Computational Mathematics, 35; Springer: Berlin, Germany, 2004.

13. Ezquerro, J.A.; Hernandez, M.A. Newton's Scheme: An Updated Approach of Kantorovich's Theory; Springer: Cham, Switzerland, 2018.

14. Grau-Sánchez, M.; Grau, À.; Noguera, M. Ostrowski type schemes for solving systems of nonlinear equations. Appl. Math. Comput. 2011, 281, 2377-2385.

15. Gutiérrez, J.M.; Magreñán, Á.A.; Romero, N. On the semilocal convergence of Newton-Kantorovich scheme under center-Lipschitz conditions. Appl. Math. Comput. 2013, 221, 79-88.

16. Hernandez, M.A.; Romero, N. On a characterization of some Newton-like schemes of $R-$ order at least three. J. Comput. Appl. Math. 2005, 183, 53-66. [CrossRef]

17. Kantorovich, L.V.; Akilov, G.P. Functional Analysis; Pergamon Press: Oxford, UK, 1982.

18. Magréñan, A.A.; Argyros, I.K.; Rainer, J.J.; Sicilia, J.A. Ball convergence of a sixth-order Newton-like scheme based on means under weak conditions. J. Math. Chem. 2018, 56, 2117-2131. [CrossRef]

19. Nashed, M.Z.; Chen, X. Convergence of Newton-like schemes for singular operator equations using outer inverses. Numer. Math. 1993, 66, 235-257. [CrossRef]

20. Ortega, J.M.; Rheinboldt, W.C. Iterative Solution of Nonlinear Equations in Several Variables; SIAM Publ.: Philadelphia, PA, USA, 2000; First published by Academic Press: New York, NY, USA; London, UK, 1997.

21. Potra, F.A.; Pták, V. Nondiscrete Induction and Iterative Processes; Research Notes in Mathematics, 103; Pitman (Advanced Publishing Program): Boston, MA, USA, 1984.

22. Verma, R. New Trends in Fractional Programming; Nova Science Publisher: New York, NY, USA, 2019.

23. Zabrejko, P.P.; Nguen, D.F. The majorant scheme in the theory of Newton-Kantorovich approximations and the Pták error estimates. Numer. Funct. Anal. Optim. 1987, 9, 671-684. [CrossRef]

24. Proinov, P.D. General local convergence theory for a class of iterative processes and its applications to Newton's scheme. $J$. Complex. 2009, 25, 38-62. [CrossRef]

25. Proinov, P.D. New general convergence theory for iterative processes and its applications to Newton-Kantorovich type theorems. J. Complex. 2010, 26, 3-42. [CrossRef]

26. Rheinboldt, W.C. An Adaptive Continuation Process of Solving Systems of Nonlinear Equations; Polish Academy of Science, Banach Center Publications; Institute of Mathematics Polish Academy of Sciences, Instytut Matematyczny Polskiej Akademii Nauk: Warszawa, Poland, 1978; Volume 3, pp. 129-142. Available online: https://eudml.org/doc/208686 (accessed on 28 November 2021).

27. Shakhno, S.M.; Iakymchuk, R.P.; Yarmola, H.P. Convergence analysis of a two step scheme for the nonlinear squares problem with decomposition of operator. J. Numer. Appl. Math. 2018, 128, 82-95.

28. Sharma, J.R.; Guha, R.K.; Sharma, R. An efficient fourth order weighted-Newton scheme for systems of nonlinear equations. Numer. Algorithms 2013, 62, 307-323. [CrossRef]

29. Soleymani, F.; Lotfi, T.; Bakhtiari, P. A multi-step class of iterative schemes for nonlinear systems. Optim. Lett. 2014, 8, 1001-1015. [CrossRef]

30. Traub, J.F.; Werschulz, A.G. Complexity and Information, Lezioni Lince; Lincei Lectures; Cambridge University Press: Cambridge, UK, 1998; pp. xii+139, ISBN 0-521-48506-1. 